\title{
WEISHEIT. HISTORISCHE DEUTUNGEN UND MODERNES BEGRIFFSVERSTÄNDNIS
}

Gehört die Frage nach der Weisheit zu den heute relevanten und beachtenswerten Themen? Die Teilnehmer(innen) einer Tagung, die von der DeutschPolnischen Gesellschaft für Philosophie im Herbst 2017 an der Universität Lodz in Zusammenarbeit mit der Schopenhauer-Gesellschaft und der Lodzener Abteilung der Polnischen Akademie der Wissenschaften veranstaltet wurde („Normativity \& Praxis: Früherer und zeitgenössischer Sinn und Begriff von Weisheit/ Past and Present Meaning and Understanding of Wisdom") haben sich mit dieser Frage intensiv beschäftigt. Sie wandten sich der Weisheitsproblematik sowohl historisch als auch systematisch zu. Drei von sieben eingereichten Beiträgen - die von Wilhelm G. Jacobs, Damir Barbarić, Leon Miodoński - behandeln vor allem die historische Relevanz des Weisheitsbegriffs. Die Rezeption der Kantischen Weisheitsauffassung bei Schelling bildet den Gegenstand von Wilhelm G. Jacobs' Aufsatz. Damir Barbarić behandelt den in Vergessenheit geratenen Begriff der Weisheit als einer nicht bloß theoretischen, sondern auch praktischen Vollkommenheit des Menschen, indem er das Besondere der Schopenhauer'schen Deutung von Weisheit durch einen Vergleich mit zwei anderen Weisheitskonzepten herausarbeitet: durch Vergleich mit einem, der an Gadamers Begriff der hermeneutischen Erfahrung orientiert ist, und einem anderen, der auf den altgriechischen Spruch „Durch Leiden gelehrt“ anspielt. Das Besondere des Schopenhauer'schen Weisheitsbegriffs steht auch im Zentrum des Interesses von Leon Miodońskis Ausführungen; Miodoński erörtert den Unterschied, der zwischen Weisheit als einer Befreiung von der Welt besteht und dem Verständnis von prudentia, die bei dem von Schopenhauer sehr geschätzten Jesuiten Baltasar Gracian zu finden ist. Ebenfalls in Anspielung an Schopenhauer als dem „,meistgelesenen Weisheitslehrer“, der aber selbst nicht allen Bedingungen erfüllt, die der Weisheit zu stellen sind, widmet sich Dieter Birnbacher dem Problem des Unterschieds zwischen Weisheit und Lebensweisheit - um auf die Frage, Was ist Weisheit?' eine Antwort zu geben.

Durch eine stärker analytische Herangehensweise sind die drei weiteren Beiträge gekennzeichnet. Guido Löhrer kündigt im Titel seines Aufsatzes eine Interpretation der sokratischen Weisheit an, was zunächst an eine historische 
Analyse denken lässt; er behandelt aber im Wesentlichen ein systematisches Problem, nämlich das des epistemischen Status des Wissens von Nicht-Wissen. Löhrers Überlegungen führen dabei zu der Schlussfolgerung, dass „,wenn Sokratische Weisheit epistemische Bescheidenheit verlangt, Sokrates nicht weise ist.“ Epistemische Aspekte der Weisheitsproblematik kommen auch in den Erwägungen von Gerhard Schönrich zur Geltung. Da man leichter zu dem Begriff einer weisen Entscheidung gelange als zu dem Begriff der Weisheit, soll sich nach Schönrich die Aufmerksamkeit darauf richten, wodurch eine Entscheidung $\mathrm{zu}$ einer weisen Entscheidung wird. Für Schönrich ist es offensichtlich, dass dazu bestimmte epistemische Leistungen des Akteurs, bestimmte MetaFähigkeiten epistemischer Art, notwendig sind. Auf die Problematik epistemischer Weisheit geht dagegen ausführlich der den Themenblock abschließende Aufsatz von Pedro Schmechtig ein. Dabei werden drei unterschiedliche Aspekte der Zuschreibung epistemischer Wahrheit - ein ontologischer, ein epistemischer und ein axiologischer Aspekt - unterschieden. In dem Aufsatz wird die These vertreten, dass die epistemische Weisheit eine „testimoniale Aktivität“" ist, womit Weisheit auf ein interpersonales Autoritätsverhältnis zurückgeführt wird.

Die gemeinsame Diskussion über Weisheit und ihre historischen und modernen Deutungen sollte auch dazu dienen, die manchmal diskursblockierenden Differenzen zwischen Anhängern einer philosophiehistorischen und einer systematischen Herangehensweise zu überwinden. Die vorliegende Publikation sowie die weiteren Aktivitäten der Deutsch-Polnischen Gesellschaft für Philosophie verstehen sich als ein kleiner Beitrag zu diesem Ziel.

Andrzej M. Kaniowski

(Lehrstuhl für Ethik, Institut für Philosophie, Universität Lodz)

Christoph Horn

(Lehrstuhl für praktische Philosophie und Philosophie der Antike,

Universität Bonn) 